
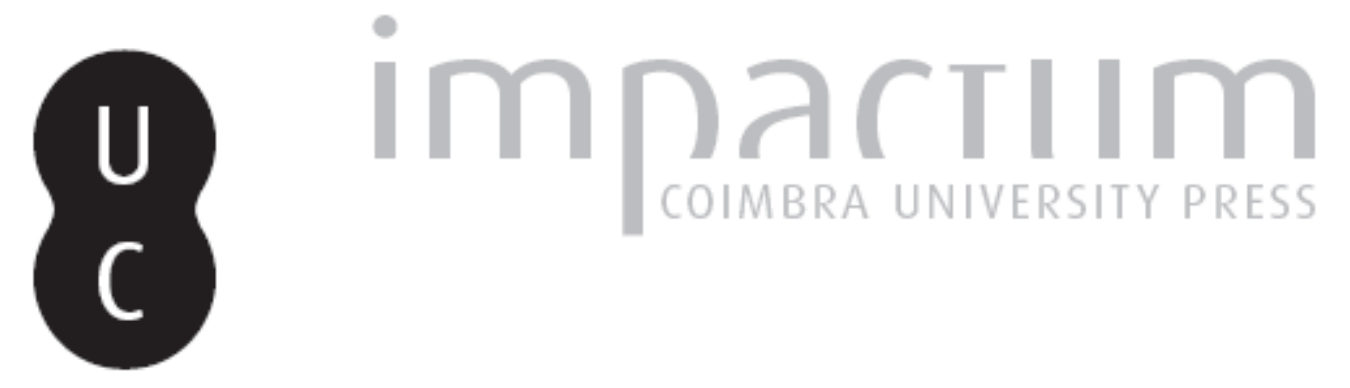

\title{
Compte rendu de J. B. Kennedy, The Musical Structure of Plato's Dialogues
}

Autor(es): Collobert, Catherine

Publicado por: Imprensa da Universidade de Coimbra

URL persistente:

URl:http://hdl.handle.net/10316.2/42174

DOI: DOI:https://doi.org/10.14195/2183-4105_12_8

Accessed : $\quad$ 26-Apr-2023 11:55:38

A navegação consulta e descarregamento dos títulos inseridos nas Bibliotecas Digitais UC Digitalis, UC Pombalina e UC Impactum, pressupõem a aceitação plena e sem reservas dos Termos e Condições de Uso destas Bibliotecas Digitais, disponíveis em https://digitalis.uc.pt/pt-pt/termos.

Conforme exposto nos referidos Termos e Condições de Uso, o descarregamento de títulos de acesso restrito requer uma licença válida de autorização devendo o utilizador aceder ao(s) documento(s) a partir de um endereço de IP da instituição detentora da supramencionada licença.

Ao utilizador é apenas permitido o descarregamento para uso pessoal, pelo que o emprego do(s) título(s) descarregado(s) para outro fim, designadamente comercial, carece de autorização do respetivo autor ou editor da obra.

Na medida em que todas as obras da UC Digitalis se encontram protegidas pelo Código do Direito de Autor e Direitos Conexos e demais legislação aplicável, toda a cópia, parcial ou total, deste documento, nos casos em que é legalmente admitida, deverá conter ou fazer-se acompanhar por este aviso.

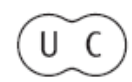


O

Established 1989

$\bigcirc$

$\vdash$

$<$

$\dashv$

ㅇ

-
$Z$
0
-
1
$Z$
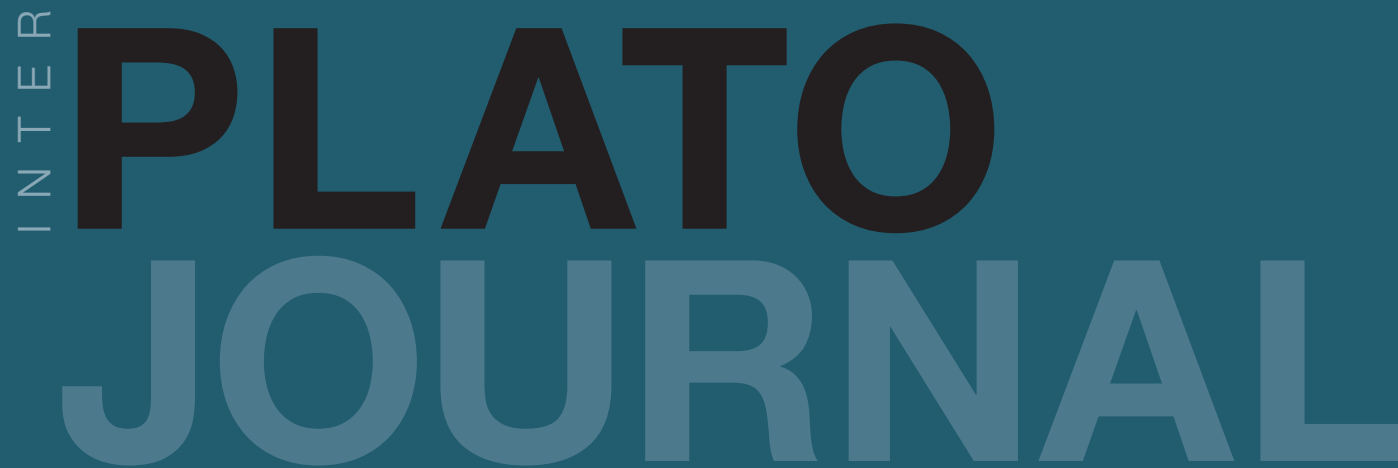

Société Platonicienne Internationale

Associazione Internazionale dei Platonisti

Sociedad Internacional de Platonistas

Internationale

Platon-Gesellschaft 


\section{Compte rendu de J. B. Kennedy, The Musical Structure of Plato's Dialogues, Durham, Acumen, 2011, 318 p.}

Cet ouvrage, composé de huit chapitres et de neuf appendices (qui contiennent des précisions utiles sur la méthode proposée), présente une thèse originale et controversée selon laquelle une structure musicale sous-tend les dialogues platoniciens, et en permet une plus riche compréhension. J. B. Kennedy s'appuie sur deux dialogues, le Banquet et l'Euthyphron pour la démontrer. Avant d'introduire sa méthodologie, il prend soin de tracer l'origine de ce type d'interprétation pour en défendre la pertinence. C'est l'objet du chapitre 1 intitulé «The nature and history of philosophical allegory». Il s'ouvre immédiatement sur l'affirmation fondamentale de l'interprétation défendue par l'auteur: «there is a musical scale embodied in each of Plato's dialogues. Symbolic passages at regular intervals are used to mark successive notes » (p. 1). C'est à une ancienne tradition d'interprétation que l'A. entend ici revenir, celle de la tradition allégoriste qu'il présente en procédant à une revue rapide de la littérature sur le sujet. L'A. doit donc défendre la possibilité que Platon allégorise et que se trouve dans les dialogues une sympathie à l'égard de la pratique de l'allégorie. Cependant, le fait que l'allégorie soit un thème des dialogues comme il est affirmé, et que les dialogues sont une forme supérieure de littérature (p. 8) ne sauraient constituer des arguments en faveur de l'allégorie : toute littérature n'est pas nécessairement allégorique et discuter même de façon récurrente d'une pratique ne signifie pas que l'on est un défenseur et un utilisateur de cette pratique. L'A. établit un lien entre la pratique du secret chez les Pythagoriciens (l'allégorie étant un moyen de masquer des doctrines qui pouvaient représenter un danger pour ses défenseurs - comme l'attestent les différentes persécutions des Pythagoriciens, des athées, et de Socrate lui-même -) et les références aux Pythagoriciens présentes dans les dialogues pour conclure au pythagorisme de Platon et en conséquence à la pratique platonicienne de l'allégorie. L'A. s'appuie ici notamment sur les travaux de Sayre (1983), Kahn $(1996,2001)$ et Dillon (2003) pour défendre l'idée d'une ontologie non écrite de nature pythagoricienne (« quasi-pythagorean ontology », p. 17). Le soutien qu'apportent ces travaux à la thèse de l'A. n'est cependant pas très clair. Les affinités que Platon peut avoir avec le pythagorisme, comme le reconnaissent ces trois auteurs, ne fait toutefois pas de Platon un pythagoricien masqué. Il faudrait ici plus d'arguments que n'en apporte l'A. et notamment un examen des divergences avec les Pythagoriciens présentes dans les dialogues. Les lectures allégoriques de Platon n'ont pas manqué d'apparaître dans l'Antiquité tardive et la Renaissance, dont certaines suggèrent l'existence de doctrines pythagoriciennes cachées dans les dialogues. Cependant, le lien entre pratique de l'allégorie et doctrines pythagoriciennes est mis à mal dans la lecture allégorique chrétienne de Platon, qui voit Platon plutôt comme l'annonciateur du message chrétien. En outre, on ne voit pas bien pourquoi il faudrait rapprocher l'existence d'une tradition ésotérique chrétienne avec la pratique de l'ésotérisme chez Platon. L'A. semble ici vouloir montrer que la

PLATO, The electronic Journal of the International Plato Society, 12, 2012.

http://gramata.univ-paris1.fr/Plato/article111.html,

(c) All rights of reproduction of any form reserved. 
pratique de l'allégorie est une pratique dominante attestée par différentes traditions ; ce qui légitimerait le fait d'inclure Platon dans cette tradition. Ce premier chapitre se termine sur une présentation des interprétations stichométriques de l'ouvrage d'Edmund Spencer, Epithalamion écrit en 1595, par A.K. Hieatt (1960) et Fowler (1964).

Le second chapitre « Introducing the dialogues' musical structure » présente la méthode utilisée pour mettre au jour la structure musicale et stichométrique des dialogues, qui montre la présence récurrente de symboles musicaux à intervalles réguliers. L'A. précise que cette structure est devenue illisible du fait de l'absence d'uniformité des lignes et des colonnes sur les copies tardives, puis avec l'édition d'Henri Estiennes (p. 32). On ne comprend cependant pas ce que l'A. veut dire ici car si cette structure était apparente, le lecteur pouvait aisément lire, sans déchiffrement intense, la doctrine soi-disant cachée.

Le symbolisme musical de Platon est, selon l'A., une caractéristique essentielle du pythagorisme de Platon. Chaque dialogue présente une gamme à douze tons qui se divisent en demi-tons et quart de tons, l'intervalle minimal. Il s'agit donc en suivant cette gamme de diviser le dialogue en douze parties ; chaque partie correspondant à un ton est divisée en quatre sous-parties (quart de ton). L'A. distingue ensuite les intervalles consonants et dissonants en fonction de sa relation à la douzième note. Les notes $3,4,6,8$ et 9 sont consonantes, 5, 7, 10 et 11 dissonantes. Les autres notes de la gamme sont plus ou moins neutres: ni consonante ni dissonante. Précisons que la gamme à douze tons n'est pas habituelle, les gammes utilisées étaient en effet à huit tons (deux tétracordes) : tel est le cas des gammes dorienne, myxolidienne, lydienne, phrygienne, etc. distinguées en fonction de la première note de la gamme et du premier intervalle ton ou demi-ton avec essentiellement trois intervalles consonants : la quarte, la quinte et l'octave (que renferme la tétraktys). Nous rencontrons une allusion à la gamme à douze tons dans un passage du Timée (35a-36b) sur la formation de l'âme du monde, que l'A. aurait pu utilement citer (qui progresse selon le facteur 3 et non 2 comme dans la gamme à huit tons). Les appendices 1 et 2 visent à défendre l'existence de la gamme à douze tons dans les dialogues. L'A. y affirme d'abord le caractère novateur de Platon en matière musicale, qui critique dans la République les théories musicales défendues par les Pythagoriciens, comme l'A. le reconnaît lui-même (p. 254). Il est dommage que l'A. n'examine pas cette dissension et ne tente pas de montrer comment elle peut se concilier avec un Platon pythagoricien. L'appendice 2 analyse des passages dans lesquels Théon de Smyrne soutient l'appartenance pythagoricienne de Platon et des passages de Thrasylle qui atteste de l'existence d'une gamme à 12 tons. L'un des deux pythagoriciens a reconnu selon l'A. l'existence d'allégories musicales chez Platon. On peut toutefois s'interroger sur la fiabilité et la crédibilité des témoins ici, qui semblent plus ou moins agir pourtant comme arguments d'autorité pour notre auteur.

PLATO, The electronic Journal of the International Plato Society, 12, 2012.

http://gramata.univ-paris1.fr/Plato/article111.html,

(C) All rights of reproduction of any form reserved. 
On aurait aimé ici plus de précisions sur la distinction entre note consonante et note dissonante dans la mesure où cette distinction est déterminante dans l'argumentation générale: «The underlying consonance and dissonance of the musical notes accounts for the peculiar structure of Plato's dialogues » (p. 38). Notes dissonantes et notes consonantes renvoient à des passages du texte qui présentent des affirmations disharmonieuses et harmonieuses. C'est ainsi selon l'A. que la structure narrative reflète la structure musicale (p. 41). Par exemple, les deux passages où Diotime fait l'éloge de la beauté se trouvent aux notes 8.0 et 9.0 (notes consonantes). À l'inverse, le passage situé à la note 7.1 (note dissonante) présente les vertus et les vices d'Éros. Il n'est pas clair ici pourquoi les vertus d'Éros sont sur une note dissonante, même si comme le soutient l'A. « the opposing elements are permanently harnessed together, but they remain an unhappy combination of positive and negative $\gg($ p. 44). Une note neutre aurait pu tout aussi bien convenir à la nature du passage, à moins que Platon ne veuille nous faire comprendre la nature essentiellement disharmonieuse d'Éros, mais ce n'est pas ce qui semble être affirmé dans ce dialogue, pas plus que dans le Phèdre.

L'A. expose ensuite sa méthode pour compter les lignes et ainsi diviser le texte. Il prend soin de préciser que l'exactitude ne saurait être absolue du fait de la corruption toujours possible du texte. Il prétend cependant pouvoir déterminer les passages corrompus grâce à la stichométrie. Le chapitre se termine sur des réponses à des objections possibles. L'A. se défend de soutenir l'existence de doctrines non écrites avec raison, puisque, selon lui, les dialogues par leur structure même contiennent des doctrines bien que non apparentes et pythagoriciennes.

Le chapitre trois «Independant lines of evidence » se propose de valider pour l'ensemble des dialogues la division en douze parties en montrant que les discours de Socrate comme de ses interlocuteurs possèdent un rapport de 12. Par exemple, le discours d'Eryximaque et d'Aristophane constituent un douzième du Banquet, celui de Socrate trois douzième. Dans le Ménéxène, le long discours de Socrate occupe les deux douzième du dialogue (p. 53). Le nombre douze joue un rôle dans l'architecture générale des dialogues et de nombreux exemples sont donnés pour illustrer ce rôle. L'A. montre en outre que des affirmations concordantes (sur la sagesse et la justice du philosophe) dans différents dialogues se trouvent aux mêmes endroits dans ces dialogues, c'est-à-dire à peu près au centre. Nous trouvons ainsi une parenté de structure, reflet d'une parenté thématique entre les dialogues.

Le chapitre quatre examine en détail la structure du Banquet et soutient la thèse originale selon laquelle certains passages de ce dialogue « contain a repeated cluster of symbols that can be understood only by comparing them to the theory of musical harmony expounded in Eryximachus' speech » (p. 62). Cette théorie repose sur la principale affirmation que la musique est l'art d'harmoniser les

PLATO, The electronic Journal of the International Plato Society, 12, 2012.

http://gramata.univ-paris1.fr/Plato/article111.html,

(C) All rights of reproduction of any form reserved. 
opposés. C'est sur la base de cette théorie que l'A. interprète le Banquet et y découvre des symboles pythagoriciens (p. 63). On aimerait ici que l'A. soit plus précis sur les symboles. Par exemple, il est possible que le terme ameletêtos ait «a symbolic import », mais en quoi est-il pythagoricien (p. 67)? Il aurait été en outre intéressant pour la thèse même de l'A. de confronter la théorie musicale proposée par Eryximaque (médecin) avec celle des pythagoriciens et montrer plus en détail que Platon fait sienne cette théorie. La mention d'un passage des Lois (664e8-a2) est trop rapide pour constituer ici un argument tout comme la réaction d'Alcibiade (p. 62-3). Le discours des symposiastes est ainsi composé de moments d'opposition et d'harmonie qui se situent respectivement à des notes et quart de ton dissonants et consonants.

Ce qui est vrai des discours l'est de l'ensemble du dialogue : les différentes voix des intervenants se trouvent harmonisées dans le Banquet (et dans tout dialogue), ce qui est l'objet du cinquième chapitre intitulé « Making the Symposium's musical structure explicit». L'A. distingue donc entre les discours harmonieux, disharmonieux et neutres : à la première catégorie appartient le discours d'Aristophane, à la seconde celui d'Alcibiade, et à la dernière le discours de Phèdre. Le discours de Pausanias qui défend un « esthétisme décadent » selon les termes de l'A. (p. 87) est légèrement dissonant. La note 2 où apparaît ce discours a pourtant été qualifiée de neutre au chapitre deux (p. 35). Mais Pausanias « platonise » (qu'on me permette l'expression) à la note 3 (note consonante) lorsqu'il affirme que la poursuite du bien est cruciale pour la vertu (p. 95). Le discours d'Agathon sur la note dissonante 5 appartient selon l'A. à la rhétorique sophistique (p.119). Il faudrait sans doute nuancer l'affirmation selon laquelle s'interroger sur la nature d'éros et de ses effets est « a sophistic jingle » (p. 118). Socrate reprendra dans son discours cette question et fera part de son accord avec Agathon sur la méthode (201d-e) — ce que souligne d'ailleurs l'A. (p. 127) —, mais le contenu sera évidemment réfuté. Le discours de Socrate qui s'étend de la note 6 à la note 9 est ainsi un mélange de consonances et de dissonances. Le discours d'Alcibiade (note 10 à 11) est dissonant à l'exception de deux passages. Il est ici curieux que le passage qui voit en Socrate un musicien soit dissonant d'autant que l'A. s'appuie sur ce passage pour soutenir son interprétation d'ensemble (p. 63). Il est vrai que Socrate est ici comparé à un joueur de flûte instrument dionysiaque associé à la démesure et condamné pour cette raison dans la République. Les images qui sont ici mobilisées ne sont-elles pas négatives qu'en apparence ? ainsi le silène qui cache une intériorité harmonieuse, cette beauté qu'Alcibiade a précisément su voir en Socrate ?

Le chapitre six consacré à l'Euthyphron défend l'existence d'une même structure musicale qui s'étend à l'ensemble des dialogues. L'A. recourt au même schéma d'analyse qui lui permet de soutenir que l'Euthyphron n'est pas un dialogue aporétique, mais qu'il s'achève sur une définition de la piété. Les éléments doctrinaux sont placés comme dans le Banquet sur des notes consonantes ( $\mathrm{p}$.

PLATO, The electronic Journal of the International Plato Society, 12, 2012.

http://gramata.univ-paris1.fr/Plato/article111.html,

(C) All rights of reproduction of any form reserved. 
221). La note 9 joue selon l'A. un rôle prépondérant et est associée au climax de l'argumentation - rôle identique dans le Banquet. De même à la note 10.3, l'A. établit un parallèle entre la demande d'Alcibiade à Socrate d'être son amant et celle de Socrate à Euthyphron qui demande à ce dernier d'être son maître. Nous avons dans les deux cas un refus de la part de celui qui est sollicité. Cependant, si la première demande est sincère, la seconde l'est sans doute moins et l'ironie de Socrate est patente.

Le chapitre sept « Extracting doctrine from structure » introduit la stichométrie pour justifier la possibilité de doctrines cachées que révèlerait la structure. Il s'attache pour cela à montrer que la doctrine du juste milieu défendue par Aristote est déjà présente chez Platon mais de manière voilée ou cachée. On trouve la présence parfois allusive de cette doctrine dans les dialogues grâce à la stichométrie. Cette présence se trouvant environ à la seconde moitié du dialogue (autour de 61\%). Cependant l'A. nous prévient que l'argument qu'il entend établir est conditionnel et qu'il faut être prudent quant à la mise au jour d'allusions dans le texte, qui ne peuvent être que probables (p. 242).

Toutefois une question demeure : si, comme l'affirme l'A. à de multiples reprises, la structure narrative reflète la structure musicale, que nous révèle de plus la structure musicale que ce qui est à «la surface » du texte ? Si en effet le texte montre clairement qu'il y a accord et harmonie qu'ajoute le fait que cet accord décelable par conséquent dans le texte soit à une note consonante ? La musique ne fait ici que refléter un contenu visible : le texte est en accord avec la musique. La musique n'exprime pas ce qui n'est pas dans le texte, autrement dit, elle ne fonctionne pas comme un supplément de sens qu'il faudrait mettre au jour et qui serait éventuellement le sens véritable uniquement accessible à ceux capables de voir. Ce qui est à la surface du texte est aussi ce qui est sous la lettre du texte : les deux coïncident. Cette coïncidence que ne manque pas de mettre en évidence l'A. tout au long de son analyse du Banquet comme de l'Euthyphron s'accorde mal (sans jeu de mots) avec l'intention première que l'A. décèle chez Platon, qui est d'introduire dans le texte des doctrines cachées.

L'A. aborde cette question au dernier chapitre qui conclut sur les implications de la structure musicale dans l'interprétation des dialogues. Ces implications sont de taille puisque nombre de problèmes épineux se trouvent ainsi résolus notamment ceux de l'anonymat et de l'intention. Platon parle de sa propre voix aux notes consonantes: «Consonant notes are associated with harmonious, positive or more Platonic ideas ». C'est ce qui permet à l'A. d'affirmer «That this passage is lodged at a dissonant note shows that its [Diotima's character] doctrines are not Platonic »). Le discours d'Aristophane, sur une note consonante, est de ce fait platonisant. Pourtant, la définition d'Éros sera réfutée par Diotime, comme l'A. le reconnait (p. 141). Cette réfutation est cruciale pour conduire à la contemplation du Beau selon Diotime (la partie de ce discours sur une note consonante est platonicien). Il est en outre affirmé que la première partie du discours de Diotime

PLATO, The electronic Journal of the International Plato Society, 12, 2012.

http://gramata.univ-paris1.fr/Plato/article111.html,

(C) All rights of reproduction of any form reserved. 
(sur une note dissonante) est anti-platonicien. Pourtant ce discours généalogique sur Éros explicite la nature du désir comme manque, qui est centrale dans les dialogues.

Le traitement de la réfutation me semble en outre problématique. Le fait que la réfutation soit toujours sur une note dissonante ne saurait indiquer dans tous les cas que Platon est en désaccord avec la réfutation menée. Ainsi lorsque Diotime réfute Agathon (note dissonante), Platon est-il en désaccord ? Il ne le semble pas, selon le traitement du discours d'Agathon par l'A. lui-même. De même Platon est-il en désaccord avec la réfutation d'Euthyphron menée par Socrate dans le dialogue éponyme ? Ici encore pas selon les analyses de l'A. La seconde question celle de l'intention est laissée en suspens, l'A. reconnaissant que des doutes légitimes demeurent (p. 247).

Le problème essentiel de la thèse générale est celui de l'existence de doctrines platoniciennes. L'A. renoue avec la tradition d'un Platon dogmatique possédant un corps de doctrines dont l'ensemble forme un platonisme pythagoricien dont on regrette cependant qu'il ne soit pas plus approfondi et justifié. Il s'agit néanmoins d'une lecture stimulantequi soulève de nombreux problèmes relativement à la question du platonisme. Ce travail mérite d'être salué pour son originalité.

Catherine Collobert (Université d'Ottawa)

PLATO, The electronic Journal of the International Plato Society, 12, 2012.

http://gramata.univ-paris1.fr/Plato/article111.html,

(C) All rights of reproduction of any form reserved. 